All this is in accordance with what almost everyone, whether or not he regards them as valid, recognizes as the standard inferences from special relativity. Then there is a chapter in which Dr Marder deals in an understanding (and, in my opinion, convincing) way with the doubts and queries expressed by a number of recent critics. Marder naturally distinguishes between the question as to whether a particular result holds good in the theoretical model and as to whether something corresponding to it holds good in the world of experience; he discusses both aspects. His next chapter is, in fact, a very useful survey of the relevant experimental evidence, inevitably with special reference to the exploitation of the Mössbauer effect. The last chapter is an introduction to some basic ideas of general relativity and their bearing upon the clock paradox. Finally there is an annotated bibliography of 241 references on the clock paradox. together with many more general items.

Dr Marder confesses that "the variety of subtle and interesting points which emerged as the work progressed came as a pleasant and welcome surprise". $\mathrm{He}$ certainly steers a clear-minded course through them all. His book will serve readers of many classes-- the general scientific reader who wants to understand what the discussion is about (without unnecessary mathematics), the teacher or student seeking material to enliven a course of relativity, the physicist interested in experimental tests of the theory, and the professional historian of science. It is an achievement

\section{Old Pharmacy}

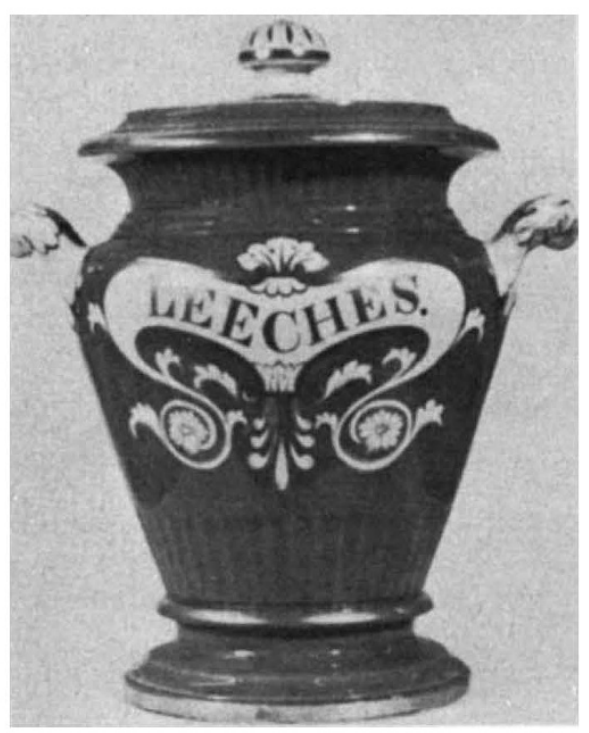

Antiques of the Pharmacy. By Leslie G. Matthews. Pp. xiv +120 . (Bell: London. June 1971.) $£ 3.50$. to provide for so many interests in a style that is seriously scientific but always concise and entertaining. To do this Marder has perforce to jump rather quickly from topic to topic; it is clear that he could very effectively enlarge upon certain of these topics in a further book of a rather different character and it is much to be hoped that he will do this also.

W. H. MCCREA

\section{Universal Mediator}

Role of Cyclic AMP in Cell Function. Edited by Paul Greengard and Erminio Costa. (Advances in Biochemical Psychopharmacology, Vol. 3.) Pp. 386. (Raven: New York, 1970. Distributed in the Eastern Hemisphere by NorthHolland, Amsterdam.) £7.35.

EVERY decade or two a metabolite appears on the biochemical scene which necessitates a reconsideration of our concepts of regulation of virtually all the major cellular functions. There is no doubt that cyclic AMP is one of these.

The Role of Cyclic AMP in Cell Function is the third volume in the recently launched serial publication Advances in Biochemical Psychopharmacology and is the outcome of a meeting held in California in February 1970. The book covers a wide area of metabolic and neurophysiological functions but, as is frequently the case with most published proceedings of conferences, the content is heterogeneous in its style. originality and interest.

The very general nature of the title is somewhat inaccurate since half of the twenty contributions concern the role of cyclic AMP in neurophysiological processes, although a serial publication on biochemical psychopharmacology would be quite justified in such a choice. It is particularly regrettable that the role of cyclic AMP in genetic transcription during catabolite repression in Escherichia coli, based on the elegant studies from the laboratories of Pastan. Zubay and Ullman and which leave no doubt as to the importance of this metabolic regulator throughout evolution, has been left out. One wonders whether some fundamental mechanism underlies the role of cyclic AMP in mediating the glucose effect in microorganisms as well as in mammalian tissues, intensely studied by Park and also not included. On the positive side, the book, in emphasizing the variety of neurophysiological functions dependent on cyclic AMP, succeeds in breaking the monotony of recent years concerning the involvement of this compound almost exclusively in hormone action.

The discovery of cyclic AMP by Sutherland, to whom this volume is dedicated, was the direct consequence of his work on the regulation of glycogen metabolism by adrenaline and glucagon, "the metabolic birthplace of cyclic AMP". Over the past decade the concept of cyclic AMP as a "second messenger" in mediating the action of many, if not all, hormones has led to some unbalanced over-generalizations on how hormones work. What this book succeeds in doing, by presenting the role of cyclic AMP in the brain and its involvement in apparently nonhormonal regulations, is to show that the regulatory function of the nucleotide transcends the importance hitherto attached to this compound exclusively as a mediator of the action of some hormones.

The book begins with two very interesting and topical contributions from the laboratories of Sutherland and Rosen on the developmental acquisition of the capacity to produce or utilize cyclic AMP. Relatively little is yet known about the function of cyclic nucleotides in differentiation so that it is a pity that the recent work on identification of 'Acrasin' as cyclic AMP and its role in aggregation patterns in slime moulds is not included. A totally reliable method for the assay of cyclic AMP in biological material is yet to come and Steiner, Parker and Kipnis, in describing their novel radioimmunoassay method, have given a brief but adequate survey of the currently used methods. Greengard and Kuo stress the pivotal role of the cyclic AMP-dependent protein kinases in explaining the multiplicity of regulatory functions of the nucleotide.

The crucial identification of "phosphorylase kinase" as the site of regulation of muscle phosphorylase by E. G. Krebs's group now offers a model for further exploration. It is most appropriate therefore that Walsh, Krebs and their colleagues have discussed such a model system as well as the detection of a specific protein inhibitor of the system, although their use of the term "receptor protein" may be arguable. Langan has also presented his work on cyclic AMP-mediated phosphorylation of histones and protamines but its real significance depends upon the eventual disentanglement of the role of histones in gene transcription in higher organisms.

The four or five articles on the role of cyclic AMP in hormone action contain a pleasantly surprising amount of new information, particularly on the action of prostaglandin presented by Butcher as well as on the pineal gland by Weiss and Crayton. Rodbell's group conclude that hormones, particularly polypeptide hormones, do not act on adenyl cyclase (or at least on its catalytic site) of the target cell but, by analogy with transistor circuits and in voking the "discriminator-transduceramplifier concept", they suggest that a 